\title{
An Effective Review of Various Routing Protocols in Wireless Sensor Network
}

\author{
Priyanka Sharma \\ GCET, Greater Noida, India
}

\begin{abstract}
Wireless Sensor Network is one of the emerging and latest research areas which are used to scan and analyze surrounding state like sound, light, pollution, temperature, wind speed, humidity, pressure, direction etc. WSNs are also used in a variety of application areas like traffic scanning, industrial scanning, machine health monitoring, traffic monitoring etc. Cluster-based WSN gives benefits, functionality, and output in numerous approaches so that multiple cluster-based routing protocols have been evolved. The aim of this work is to give the short study of the clustering based routing protocols. This paper explains the introduction of the cluster based routing techniques in Static WSN and in Mobile WSN. The major concern of this paper is to examine two dissimilar hierarchal cluster-based routing protocols such as homogeneous and heterogeneous.
\end{abstract}

Keywords: Wireless sensor network, LEACH, Sensor node, Cluster head, MWSN, SWSN, Routing protocol

\section{Introduction}

Wireless Sensor Network is one of the latest technologies used nowadays. It is composed of distributed and selfdetermining little, a small battery powered sensor nodes which are used to feel, operate, enumerate, and interface with each other [1]. A sensor network is employed in unspecified geographical extents that are made up of a wide range of small nodes. The fundamental operations which are used to execute in WSN may disrepair the battery of sensor nodes rapidly, because of which the craved for operations remain incomplete and this results in a network failure. To keep away such circumstances, the main focus shifted towards the battery life of the sensor nodes. It is important to know the components that influence the life of the battery and ways to minimize the consumption of energy which is one of the main provocations [5]. Cluster Based Routing is used in both mobile as well as in static WSN. In this sensors are assembled into dissimilar clusters which consist, Cluster Head and gather information from each node in its cluster. In this paper, WSN is classified as Static Wireless Sensor Network (SWSN) and Mobile Wireless Sensor Network (MWSN) [6]. The SWSN are composed of sensor nodes that are static in nature i.e. the place of the nodes are fixed Whereas MWSN composed of sensor nodes which are static in nature i.e. the place of the nodes are not fixed, they can easily move place to place.

To please the requirements of an application, the physical deployment of the WSN need to be review. The network deployment recognizes the network density [2]. The level of redundancy is not exactly similar to the whole network also dense zone and sparse zone both have a different effect on sleeping decision. When the sensing coverage is lower than the precision level is higher. The random deployment has a dissimilar place for nodes. Thus, the node which is used for joining two categories of the network will always in an active position to minimize. The place of a node in WSN defines its role and operations [4].

The radio communication is the main part of power consumption. When the transmission is higher than the life of sensor node is shorter [7]. Several methods are known to calculate the stored information, future information and remove the redundancy [3].

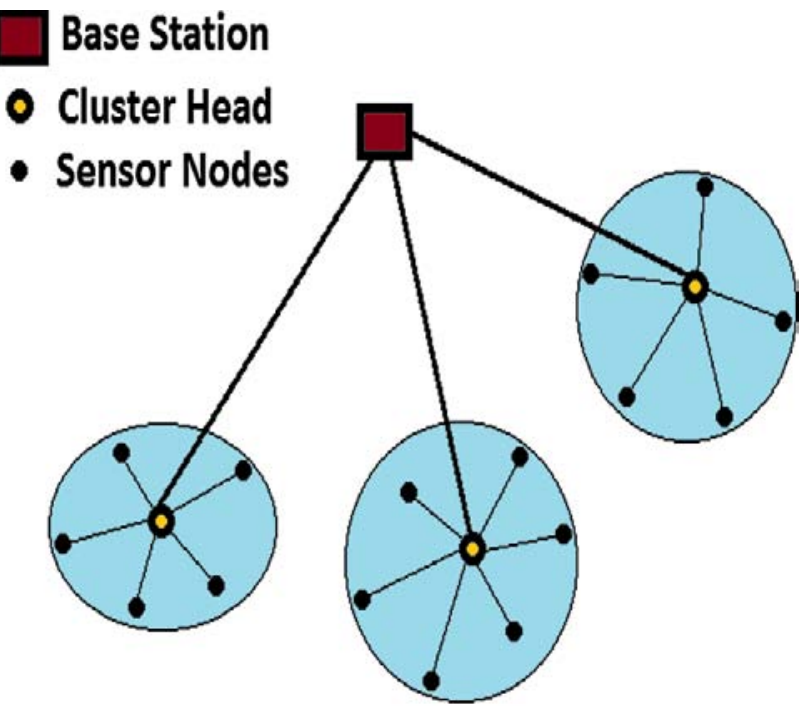

Figure 1: Wireless Sensor Network

Impact of network deployment on data credibility

Numerous points are there in network deployment that is used to amount the entire scanned region. Some of these points are as follows [10]:

- Mainly the deployment model: In a small region, the sensor node is positioned one after one to ensure a scanned region. However, in a large region, the sensor nodes are deployed randomly.

- The network density: The numbers are represented as per square meter in the sensor node. This point is swiftly handled by positioned nodes. But it is hard to handle randomly deployed WSNs.

- The sensing coverage per sensor node: circle is used for representing each node that is placed in the middle of it with a radius $r$ described by the developer of the system. 


\section{International Journal of Science and Research (IJSR) \\ ISSN (Online): 2319-7064}

Index Copernicus Value (2015): 78.96 | Impact Factor (2015): 6.391

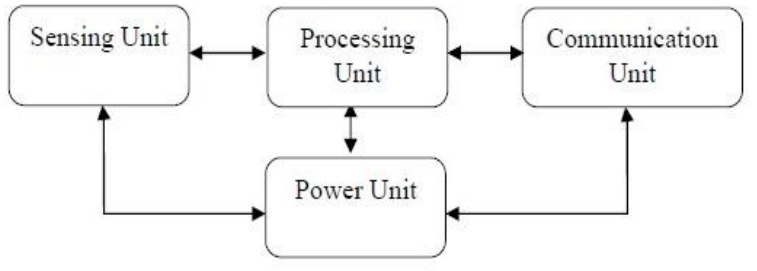

Figure 2: Block diagram of sensor node

\section{Sensor Nodes' Characteristics}

i) Sensor nodes' types: a classification by application nature

- Terrestrial Sensor Nodes could be deployed for large-scale environmental applications like a desert, forest etc.

- In industrial applications, such as underground mine or petroleum fields, strong sensor nodes are required

- The Underground sensor nodes (UGSN) have restricted because their battery is unchangeable or not chargeable.

- The Underwater Sensor Nodes (UWSN) are planned to be deployed in underwater applications

- Multimedia application is another type of sensor node which is also known as MSNs (Multimedia Sensor Nodes).

- TSNs, UGSNs, UWSNs and the MSNs which are mobile and fixed can be used in several militaries, civil and industrial applications.

ii) Technologies advancements

- The first one is a basic, light event-based operating system which is written in nesC

- The second is a sunspot which is a product of Sun Microsystems, Inc. encompassing both hardware and software Sun.

iii) Radio entity: importance and power consumption

The radio communication is the main organization of the sensor node to make the wireless network. This organization is familiar to be the main consumer of power [8].

\section{Cluster-Based Routing Protocols in WSN}

There are two types of schemes in clusters protocols: Homogeneous schemes and Heterogeneous schemes.

\subsection{Homogeneous schemes}

Homogeneous schemes have an equal amount of energy levels. LEACH, HEED, PEGASIS and much more come under homogeneous schemes, where LEACH is a basic protocol. LEACH protocol is a self-organized and selfadaptive protocol that represents Hierarchical routing [9, 11]. LEACH protocol has two phases: Setup phase is the phase in which cluster heads are chosen, and Steady-State phase is a phase in which cluster head is maintained.

\subsection{Heterogeneous Schemes}

Heterogeneous schemes have a different amount of energy levels and surroundings [9, 12]. E-LEACH, DEEC, SEP, EECS, Novel HRP and much more come under Heterogeneous schemes. M-LEACH Protocol gives mobility to cluster head and non-cluster heads node. are small, with limiting processing and computing resources, and they are inexpensive compared to traditional sensor maintaining the integrity of the specifications

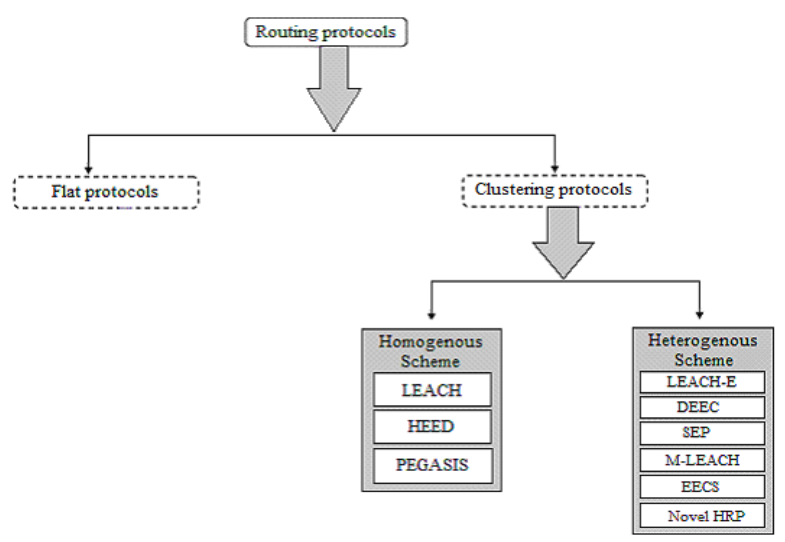

Figure 3: Cluster-Based Routing Protocols in SWSN

\section{Cluster-Based Routing Protocols in SWSN}

i) LEACH chooses node as cluster heads is a cluster-based protocol. The operation of LEACH protocol is performed in two phase: set-up phase is a phase in which clusters are established and Cluster Head is chosen for every cluster and steady phase is a phase in which TDMA nodes transfer information to each cluster head under the time which is allocated.

ii) Hierarchical Cluster-based Routing protocol (HCR) is the one in which each cluster uses round-robin technique and is supervised by a headset. A cluster member is used to transmitting notes to the cluster head and then gathers the notes and transmits it to the Base Station (BS) [9]. The simulation is executed on versions of HCR (HCR-1, HCR-2) and LEACH and discovered that HCR-1 manifest a little enhancement over LEACH but for HCR-2 the enhancement is an increase [8].

iii) In cluster-based energy-efficient routing protocol, the sensor nodes do not know their position. As claimed by the state of the network, it utilizes the left out the energy of sensor networks and desire number of CHs. It was discovered that the data rate and life of the sensor networks of this protocol is enhanced as compared to LEACH.

iv) Dynamic Clustering Reactive Routing (DCRR) is a protocol that depends upon the structure and the fundamentals of neural network where the sensor nodes are event driven [9]. The execution of DCRR is contrasted with TEEN and discovered that DCRR performs superior in respect to battery power distribution and enhanced the effectiveness of energy and life of the network.

v) Threshold sensitive Energy Efficient sensor Network (TEEN) protocol relies on LEACH routing protocol which is used in WSN for time critical approaches [14]. It contains nodes with first level and second level $\mathrm{CHs}$ which are formed away from BS and near to BS. There are two categories of neighbor nodes i.e. is Hard Threshold (HT) mode and Soft Threshold (ST) mode. 


\section{International Journal of Science and Research (IJSR) \\ ISSN (Online): 2319-7064}

Index Copernicus Value (2015): 78.96 | Impact Factor (2015): 6.391

\section{Cluster-Based Routing Protocols in MWSN}

i) Cluster Based Routing (CBR) protocol is the protocol which is performed by using MATLAB and it minimizes the loss of packets by $25 \%$ as compared to LEACH protocol [13]. There is an enhancement in transferring information as contrasted with the LEACH-mobile protocol.

ii) Shortest Path (SP) routing protocol is a protocol which minimizes the cost of establishment of WSN and the complications of saving energy. The output discovers that Shortest Path routing protocol performs as compared with LEACH by defining again the task of nodes, by keeping sleeping mode on for some nodes and transferring data packets from shortest path to the sink [7].

iii) Energy-efficient Chain-cluster Routing protocol (ECRM). In this the nodes which are static are hard to recharged battery, are put into the transmission backbone to maintain the network connection and enhanced the efficiency of energy.

\section{Conclusion}

WSN contain self-ruling, the restricted power of battery, less value sensor nodes install in a particular region. The most important approach used for data routing is cluster based routing. Efficient clustering system is used by which efficient energy is attained to lesser the use of energy. In the last few years, routing in wireless sensor networks has pulled a great deal of care and has proposed unique challenges in comparison of conventional data routing in wired networks. The goal of this thesis is to analyze and calculate variety of routing protocols in WSNs and examine their primary routing selection principles.

\section{References}

[1] S.K. Singh, M.P. Singh, and D.K. Singh, "A survey of Energy-Efficient Hierarchical Cluster-based Routing in Wireless Sensor Networks", International Journal of Advanced Networking and Application (IJANA), Sept.Oct. 2010, vol. 02, issue 02, pp. 570-580.

[2] Jun Zheng and Abbas Jamalipour, "Wireless Sensor Networks: A Networking Perspective", a book published by A John \& Sons, Inc, and IEEEE, 2009.

[3] W.R. Heinzelman, A. Chandrakasan, and H. Balakrishnan, "Energy-efficient Communication Protocol for Wireless microsensor Networks", in IEEE Computer Society Proceedings of the Thirty Third Hawaii International Conference on System Sciences (HICSS '00), Washington, DC, USA, Jan. 2000, vol. 8, pp. 8020.

[4] Bhoopathy, V. and R.M.S. Parvathi, "Securing Node Capture Attacks for Hierarchical Data Aggregation in Wireless Sensor Networks", International Journal of Engineering Research and Applications (IJERA), Vol. 2, Issue 2, pp.466-474,Mar-Apr 2012.

[5] Karl Holger, Willig Andreas, "Protocol and Architecture for Wireless Sensor Network", John Willey and Sons Ltd, 2005

[6] Amrinder Kaur, Sunil Saini," Simulation of Low Energy Adaptive Clustering Hierarchy Protocol for
Wireless Sensor Network," International Journal of Advanced Research in Computer Science and Software Engineering, Volume 3, Issue 7, July 2013.

[7] I.F. Akyildiz, W. Su, et al., "Wireless sensor networks: a survey", Computer Networks 38 (4) (2002) 393-422.

[8] Akyildiz, I. F., Melodia, T. \& Chowdhury, K. R. (2007). A survey on wireless multimedia sensor networks, Comput. Netw. 51(4): 921-960.

[9] K.SOHRABY,D.MINOLI,T.z NATI" WIRELESS SENSOR NETWORKS, Technology, Protocols, and Applications" Published by John Wiley \& Sons, Inc., Hoboken, New Jersey. 2007

[10]L. Sun, J. Li, Y. Chen, et al., "Wireless Sensor Network", Tsinghua University Press, Beijing, China, 2005.

[11] J. Yick, B. Mukherjee, and D. Ghosal, "Wireless sensor network survey", Computer Networks, Vol. 52, No. 12, 2292-2330, 2008.

[12] Basavaraj S. Mathapati , Patil Dr. Siddarama. R. , and Mytri Dr. V D. "Energy Efficient Cluster based Mobility Prediction for Wireless Sensor Networks", (IEEE (International Conference on Circuits, Power and Computing Technologies (ICCPCT)), 2013.

[13] Sachin Gajjar, Shrikant N. Pradhan, Kankar Dasgupta, "Performance Analysis of Cross Layer Protocols for Wireless Sensor Networks",

[14] Houda Zeghilet, Nadjib Badache, and Moufida Maimour, "Energy Efficient Cluster-based Routing in Wireless Sensor Networks", IEEE symposium on computers and communication 2009, ISCC- 2009, pp. 701-704, sepember 2009. 